\title{
IVH-FL: Intelligent Vertical Handoff based on Fuzzy Logic Decision
}

\author{
Rushdi A. Hamamreh ${ }^{1}$ \\ 1 Assoc.,Professor at the Computer Engineering Department, Al-Quds University, rushdi@staff.alquds.edu, Palestine.
}

\begin{abstract}
The most important issue in integrated next generation wireless systems (NGWS) by fourth generation networks which consists of various wireless architectures extending from cellular networks to satellite networks is to allow everyone around the world to connect seamlessly to applications anywhere at any time through the best network. Heterogeneous networks have created many challenges such as mobility management, handoff, resource management. For always best connectivity the selection of parameters plays an important role in the decision of vertical handoff, some of parameters are depend upon MT and some are depend upon the network conditions . In this paper we designed Intelligent Vertical Handoff based on Fuzzy Logic Decision model ( IVH-FL), IVH-FL model which has five parameters for vertical handoff decision : Received Signal Strength (RSS), Available bandwidth (B), Users Preference (UP), Mobile Speed (SM) and Power Consumption (PC), with the help of Fuzzy Logic tool box and concept of fuzzy linguistic variables. The results confirmed improvement performance, and reduced the number of unnecessary handover .
\end{abstract}

Keywords: Vertical Handoff; Fuzzy Logic; wireless systems; IVH-FL.

\section{INTRODUCTION}

Next generation wireless network (NGWS) is a network of emerging different architectures ranging from cellular to satellite networks. This technology is a very complex technology of integrating different new techniques, and services for a higher speed wireless internet access. This integration invokes some challenges, such as handover decision making and mobility management. Mobility is the most important feature of today's wireless networking system. Mobility can be attained by handoff mechanisms in wireless networks. Handoff management is one of the components of mobility management which controls the change of mobile terminal's point of attachment during active communication. Handoff is the process of changing the channel connection when the call is in progress. Handoff can be divided into two different types - horizontal and vertical. The switching between points of attachment or base stations that belong to the same network is called Horizontal handoff and this is required in homogeneous networks. The switching between points of attachment or base stations that belong to the different network technologies is called Vertical handoff and this is required in heterogeneous networks. The process of The vertical handoff process involves three main phases $[4,5,13,14]$, namely system discovery, vertical handoff decision, and Vertical handover execution. During the system discovery phase, the mobile terminal determines which networks can be used. These networks may also advertise the supported data rates and Quality of Service (QoS) parameters. In the Vertical handover decision phase, the mobile terminal determines whether the connections should continue using the current network or be switched to another network. The decision may depend on various parameters or metrics including the type of the application, minimum bandwidth and delay required by the application, access cost; transmit power, and the user's preferences. During the Vertical handover execution phase, the connections in the mobile terminal are re-routed from the existing network to the new network in a seamless manner. This phase also includes the authentication, authorization, and transfer of a user's context information [6]. Handoff management aims at controlling the change of an access point (AP) in order to maintain the connection with the moving device during the active data transmission. The problem is exacerbated by the presence of APs adopting different technologies. Hence vertical handoffs, that is, handoff procedures between APs of heterogeneous technology, should be taken into account [7].

Traditional handoff process, called horizontal handoff, takes place to provide a seamless service when a user moves between two adjacent cells. Generally, horizontal handoff is based on only single parameter such as RSS (Received Signal Strength), SNR (Signal-to- Noise Ratio), etc. and initializes when the received signal strength indicator (RSSI) or SNR drops below a specified handoff threshold. It is also performed between two access points each of which has the same network technology. On the other hand, the vertical handoff process takes place between different technologies. Because the next generation wireless systems involve a mixture of various technologies, it is obvious that traditional handoff mechanisms will not be sufficient. Using only one metric for vertical handoff decision making is not efficient since existing networks overlap with each other. Considering more performance metrics, such as; data rate, monetary cost, user's speed, and etc., as well as RSS more realistic performance results can be achieved for these heterogeneous structures. In general, the vertical handoff 
process is divided into three steps. First, a mobile node must know which wireless systems are reachable. This step is called system discovery. The next step is a handoff decision, in which the mobile node evaluates vertical handoff parameters associated with a new wireless system (network) to make handoff decision. If the mobile node decides to handoff to other network, the last step will proceed.

The last step is handoff execution. If the mobile node decides to perform vertical handoff, it executes the vertical handoff procedure to be associated with a new wireless system.

Note that a handoff execution means a successful handoff to the another network. Consequently, in heterogeneous wireless networks, it is critical to design an efficient vertical handoff algorithm for providing seamless connection. Artificial intelligent algorithms based on fuzzy logics are some of the approaches to solve vertical handoff problems, in this paper five parameters are considered for vertical handover which are RSS, bandwidth ,Users Preference , Mobile Speed and Power Consumption .By using IVH-FL model, this new proposed model is expected to generate many challenges for heterogeneous networks, especially in the design and implementation of network infrastructures, and the most important challenge is in vertical handover when one user can change his access network without the loss of services.

\section{RELATED WORKS}

We note From the literature surveyed, authors used different terms such as models, techniques and approaches to refer mechanisms. In order to compile vertical handover mechanism for $4 \mathrm{G}$ wireless networks, it is essential to study existing VHO mechanisms because it will assist in the identification of requirements for VHO mechanism and design Intelligent Vertical Handoff based on Fuzzy Logic Decision algorithm. In this survey we are interested with kinds of approaches that are using artificial intelligence techniques, combining several parameters such as network conditions and Mobile Terminal's (MT) mobility in the handoff decision. Some of research works have been done and have various types of techniques and algorithms have been introduced for vertical handover to improve the QoS :

- Ali Çalhan \& Celal Çeken developed an adaptive fuzzy based handoff decision system which combines parameters such as data rate, cost and RSSI in order to get training elements to ANFIS. With the training element of ANFIS from a fuzzy based system, the rules and the membership function can be properly tuned to optimize the handoff performance[4].

- In [5] Manoj Sharma \& Dr. R.K.Khola proposed an intelligent approach to find out the vertical handover decision in a multi network environment. The Sugeno Fuzzy Inference system is used to find the decision for vertical handover. The inference uses the crisp input values for network parameters such as available bandwidth, network load and signal strength. The value of these network parameters are generated by the event generator and are fed fuzzy inference system. The output of the fuzzy system is handover decision.

- Manoj Sharma \& Ram Kishan Khola proposed an algorithm to process the handover decision in heterogeneous wireless networks. Fuzzy normalization not only includes the advantage of imprecise data dealing, but also enables the comparison of different parameters in heterogeneous networks. It can make choosing a NAP easier and raise performance [6].

- In [10] Zhang presents an algorithm that uses fuzzy logic combined with some classical Multi Attribute Decision Making (MADM) methods, Simple Additive Weighting (SAW) and Technique for ordering preferences by similarity to ideal solution (TOPSIS), for handover decision in order to combine and evaluate multiple criteria simultaneously.

- Song and Jamalipour in [11] explain an algorithm that combines the analytic hierarchy process (AHP) and the grey relational analysis (GRA) methods to decide the best network for mobile users through finding the tradeoff among service application, network condition, and user's preference.

- Yang and $\mathrm{Wu}$, their proposal uses fuzzy logic and MADM; the proposed Algorithm is divided into four parts: traffic classification, resource estimation for reservation, admission control and RAT selection [12] .

\section{Proposed IVH-FL model}

In order to design Intelligent Vertical Handoff based on Fuzzy Logic Decision algorithm for next generation wireless networks, it is essential to study the identification of important five parameters for proposed IVH-FL.

\subsection{Input parameters for proposed model}

In this paper five parameters have been studied. Some of the parameters are dynamic requirements including RSS, mobile speed, and user preferences, non-dynamic requirements include power consumption, and bandwidth as parameters. A good handoff mechanism decision model should have both dynamic and non-dynamic metrics. We will introduce the parameters for the proposed model as shown:

\section{Available Bandwidth (B)}

Bandwidth is a measure of the width of a range of frequencies. It is the difference between the upper and lower frequencies in a contiguous set of frequencies. In order to provide seamless handoff for Quality of service (Qos) in 
wireless environment, there is a need to manage bandwidth requirement of mobile node during movement. Bandwidth is generally known as the link capacity in a network. Higher offered bandwidth ensures lower call dropping and call blocking probabilities.

\section{Speed of Mobile(SM)}

It is the speed at which the Mobile Terminal (MT) is moving. In vertical handoff algorithms, the speed factor has a larger and important decision binding effect than traditional handoff decision algorithms horizontal handoff. When the users travel at high speed within a network coverage area is discouraged the idea to initiate vertical handoff process because after a short period of time the user will have to go back to the initial network because it will get out from under cover network host.

3. Received Signal Strength (RSS)

The performance of a wireless network connection depends in part on signal strength. Between a mobile node (MN) and access point (AP), the wireless signal strength in each direction determines the total amount of network bandwidth available along that connection. RSS is an important criterion for proposed algorithms as well. It is the strength of the signal received, as the RSS of the neighboring network rises above the threshold the Vertical Handoff is. RSS is easy to measure and it is directly related to the services quality and there is a relationship between the RSS reading and the distance from the mobile terminal to its point of attachment.

4. Power Consumption (PC)

In integrated next generation wireless systems networks, we need to improve energy efficiency. Power is not only consumed by the user terminal but also attributed to base station equipment. Power is also consumed during mobile switching or handoffs. During handoff, frequent interface activation can cause considerable battery drainage. The issue of power saving also arises in network discovery because unnecessary interface activation can increase power consumption. It is also important to incorporate power consumption factor during handoff decisions.

\section{User Preferences (UP)}

When handover happens, the users have more options for heterogeneous networks according to their preferences and network performance parameters. The user preferences could be preferred networks, user application requirements (real time, non-real time), service types (Voice, data, video), Quality of service (It is a set of technologies for managing network traffic in a cost effective manner to enhance user experiences for wireless environments) etc. User Preferences can also be considered for $\mathrm{VHO}$ in $4 \mathrm{G}$ wireless networks.

\subsection{Fuzzy inference system (FIS) for IVH-FL}

The advantage of fuzzy logic based algorithm is the ability to take multiple parameters into account and give the best possible solution for handoff decision, especially when the nature of the problem exhibits uncertainty. The Fuzzy inference system is based on the concept of fuzzy set theory, fuzzy reasoning and IF-THEN rulers. The fuzzy inference engine is based on the Sugeno fuzzy inference system, whose computational performance is more efficient than the Mamdani system. Intelligent Vertical Handoff based on Fuzzy Logic Decision algorithm can be applied to the handoffs initiated by mobile nodes (MNs). In such situations, fuzzy logic based handover is used to select an optimized network for the MN. Figure 1 shows the FIS contains number of functional blocks:

1. Fuzzifier - The fuzzifier transforms the crisp inputs into linguistic values. The fuzzifier maps a crisp point, $\mathrm{x}=$ $[R S S=x 1, B=x 2, U P=x 3, B L=x 4 \& N C=x 5] T \in U$, into a fuzzy set $A=\{(R S S, \mu A(R S S)),(B, \mu A(B)), \cdots,(x n$, $\mu \mathrm{A}(\mathrm{xn}))\}$ in $\mathrm{U}$, where $\mu \mathrm{A}: \mathrm{U} \rightarrow[0,1]$ is the membership function of the fuzzy set $\mathrm{A}$ and $\mu \mathrm{A}(\mathrm{xi})$ is the membership degree of xi in A.

2. Fuzzy rule database - It contains a number of fuzzy IF-THEN rules that have been useful in decision making criteria for handoff. If a fuzzy system has $\mathrm{n}$ inputs and a single output which is called in the proposed model handoverdecision, its fuzzy rule $\mathrm{Rj}$ is of the general form: IF $\mathrm{x} 1$ is $\mathrm{A} 1 \mathrm{j}$ and $\mathrm{x} 2 \mathrm{is} \mathrm{A} 2 \mathrm{j}$ and ... and $\mathrm{xn}$ is Anj, THEN y is $\mathrm{Bj}$.

3. Fuzzy inference engine - The fuzzy inference engine performs the inference operations on the fuzzy rules. Fuzzy logic principles are used to combine fuzzy IF-THEN rules in the rule base, and fuzzy sets in $\mathrm{U}=\mathrm{U} 1 \times \mathrm{U} 2 \times \ldots \times \mathrm{Un}$ are mapped into fuzzy sets in $\mathrm{V}$. Let $\mathrm{A} \in \mathrm{U}$ be the input to the fuzzy inference engine, and let the fuzzy rule be represented as the fuzzy implication $\mathrm{R}=\mathrm{R} 1 \mathrm{j} \times \mathrm{R} 2 \mathrm{j} \times \mathrm{R} 3 \mathrm{j} \times \cdots \times \mathrm{Rnj} \rightarrow \mathrm{Gj}=\mathrm{C}$ in $\mathrm{U} \times \mathrm{V}$. Then, each fuzzy IFTHEN rule determines a fuzzy set $\mathrm{D}$.

4. Defuzzifier - A defuzzifier transforms the fuzzy results of the inference into a crisp output. It maps fuzzy sets in V into a crisp output $y \in \mathrm{V}$. In this paper sugeno types FIS is used because it gives better performance than mamadani FIS. In this paper five parameters has been proposed some of them depend upon the network and the others depend on mobile (MT). 
Figure 1. FIS of the proposed IVH-FL.

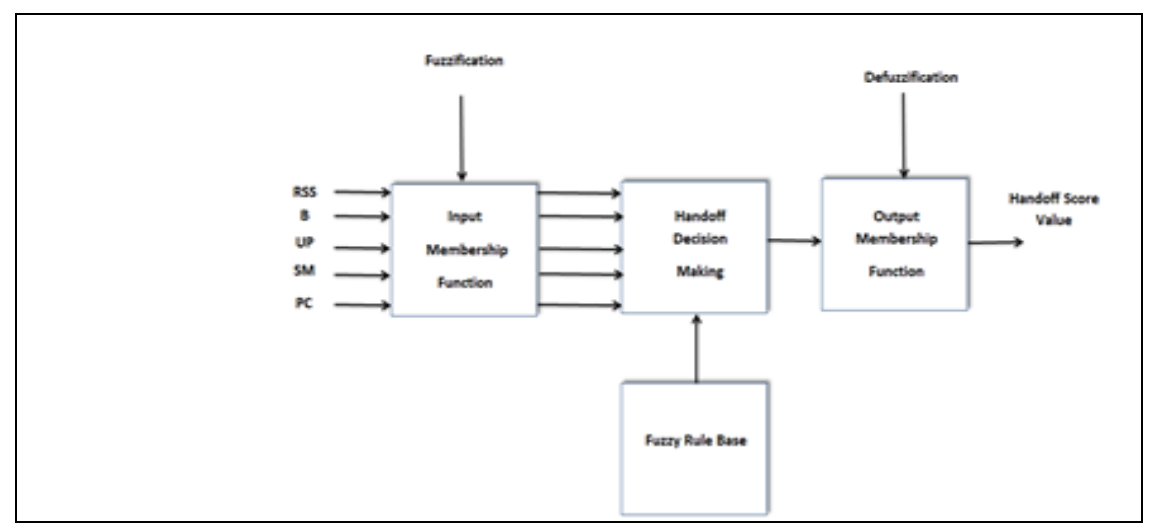

In order to compare the attributes of different values and different units of measurement it is necessary to use the process of normalization. Normalization is needed to ensure that the values in different units are meaningful as in equation(1) below:

$$
\mathrm{N}(\mathrm{x})=\frac{\mathbf{X}-\mathbf{X x}_{\mathrm{min}}}{\mathbf{X}_{\max }-\mathbf{X}_{\min }}
$$

Where $\mathrm{x}=\mathrm{RSS}, \mathrm{B}, \mathrm{UP}, \mathrm{SM} \& \mathrm{PC}$. In (1), RSS, B and UP denote the original values; N (RSS), N (B), N (UP), N (SM) and N (PC) are the normalized values.

After the inputs have been collected originally, they are mapped into the normalized values by the normalized functions (1). Each of the normalized input parameters is assigned to one of three fuzzy sets by membership functions. The membership functions (MFs) of RSS, B, and UP ,SM and PC are shown in figures 2-6.

Figure 2. Membership function for input variable Received Signal Strength (RSS).

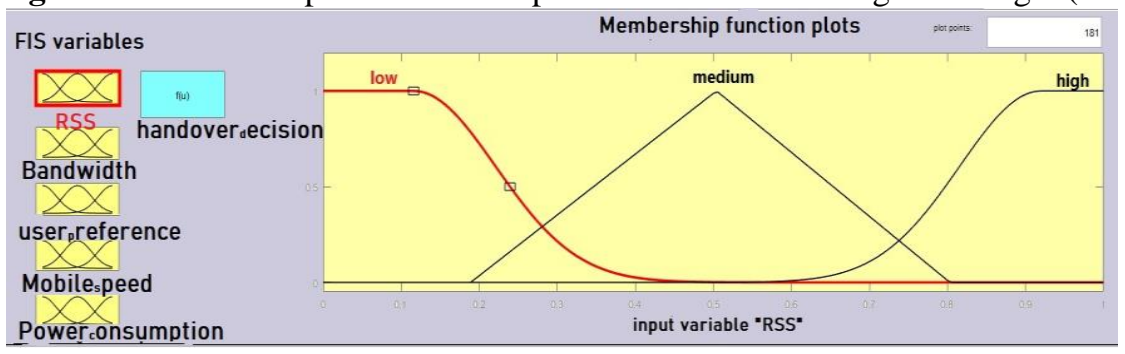

Figure 3. Membership Function for input variable Bandwidth (B).

FIS variables
Mobilespeed
Powerconsumbtion

Figure 4. Membership Function for input variable User Preference (UP).

FIS variables
Mobilespeed
Poweronsumption


Figure 5. Membership Function for input variable Speed of Mobile (SM).

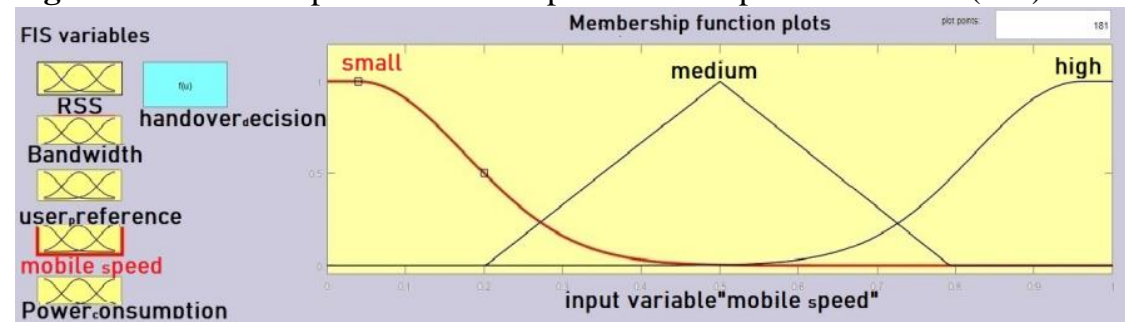

Figure 6. Membership Function for input variable Power Consumption (PC)

Mobilespeed
Dower consumption
user reterence

The output of the fuzzy system is handover decision which is based on the weight vector for each of the input parameters and membership function of each parameter, figures 7 and 8 the output membership function (very low, low ,medium ,high and very high) .

Figure 7. Membership Function for output variable handover-decision.

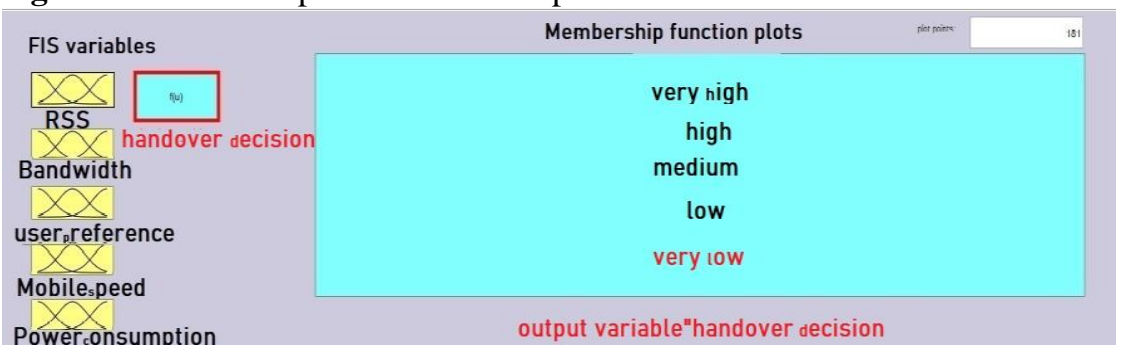

Figure 8. IF-THEN fuzzy rules viewer

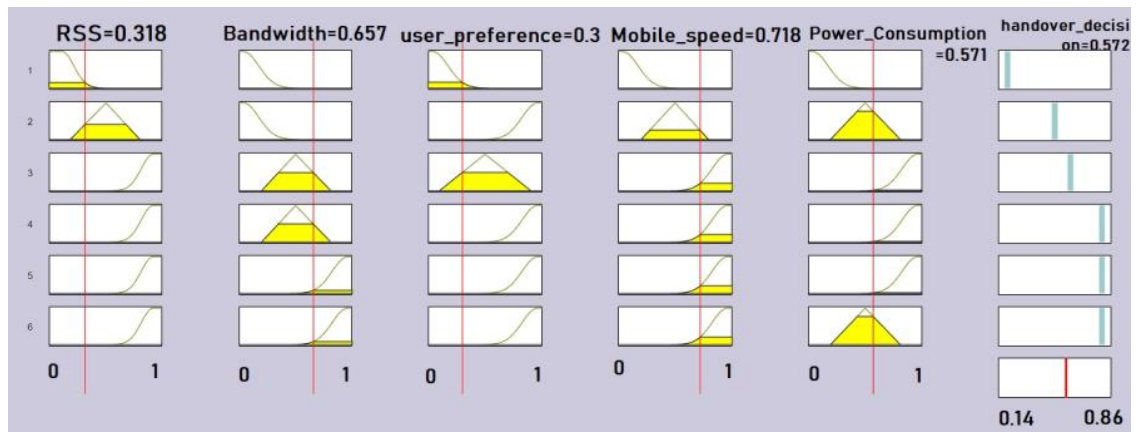

\section{Result and Discussion}

On the basis of five parameters fuzzy rules are defined and a decision algorithm is performed with the help of fuzzy tools . The fuzzy inference engine is based on the Sugeno fuzzy inference system, whose computational performance is more efficient than the Mamdani system. The number of rules for five parameters according to expression used in FIS can be calculated as: $[3] 5=243$

Here 3 are the number of membership functions.The output range function is from 0 to 1 , the vertical handoff decision is based on the range of output. The output membership function in surgeon constant. 
Some of The IF-THEN rules are given as:

1. If (RSSI is low) and (Bandwidth is small) and (user_preference is negative) and (Mobile_Speed is small) and (Power_Consumption is low) then (handover_decision is very_low).

2. If (RSSI is medium) and (Bandwidth is small) and (user_preference is positive) and (Mobile_Speed is medium) and (Power_Consumption is medium) then (handover_decision is medium) .

3. If (RSSI is high) and (Bandwidth is medium) and (user_preference is zero) and (Mobile_Speed is high) and (Power_Consumption is high) then (handover_decision is high).

4. If (RSSI is high) and (Bandwidth is medium) and (user_preference is positive) and (Mobile_Speed is

5. high) and (Power_Consumption is high) then (handover_decision is very_high) .

6. If (RSSI is high) and (Bandwidth is large) and (user_preference is positive) and (Mobile_Speed is high) and (Power_Consumption is high) then (handover_decision is very_high) .

7. If (RSSI is high) and (Bandwidth is large) and (user_preference is positive) and (Mobile_Speed is high) and (Power_Consumption is medium) then (handover_decision is very_high).

The IVH-FL handoff decision scheme can be given as:

a) If the handoff decision output is greater than 0.7, then the MN chooses WLAN. If the current access network is WWAN, then the MN makes a handoff; otherwise, no handoff.

b) If the handoff decision output is between .45 to .7 then the $\mathrm{MN}$ stays in the current access network. This could reduce the unnecessary handoffs.

c) If the handoff decision output is less than 0.45 , then the MN chooses WWAN. If the current access network is WLAN then the MN makes a handoff; otherwise, no handoff .

Figure 10: shows a surface viewer based on the Sugeno fuzzy inference system.

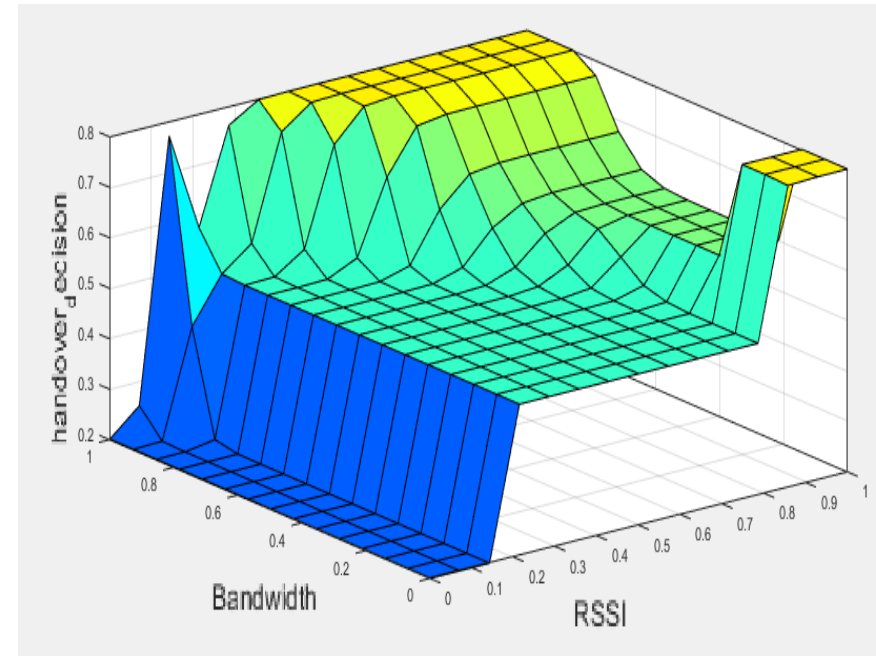

\section{Conclusion}

In this paper , a new Intelligent Vertical Handoff based on Fuzzy Logic Decision algorithm is introduced based on the known of five parameters including RSS, Bandwidth, users preference, Speed of Mobile and Power Consumption. For getting more accurate decision system handover we used fuzzy logic which considers intelligent behavior to cope with uncertainty . For the handoffs initiated by MN s, IVH-FL is employed to select the most appropriate network for the MNs. Afterward, the selected MNs are handed over to other nearby BSs. The results show the accurate IVH-FL model, help to balance the network resources and improve the performance of the networks. our proposed solution concludes in the following steps:

1. Design an Intelligent Vertical Handoff based on Fuzzy Logic Decision algorithm based on the concept of fuzzy linguistic variables to cope with requirements of the heterogeneous wireless network.

2. This review suggests that improvements in performance be achieved when using these parameters for IVH-FL decision handover. 
3. Develop an intelligent handover decision with the most five requirements for handoff using artificial intelligence behavior to handle uncertainty for a dynamic mobile environment .

4. The most advantages of our proposed scheme are to reduce blocking rate and achieve high throughput with low latency.

\section{REFERENCES}

[1] Nikolaos Psimogiannos, Aggeliki Sgora, Dimitrios D. Vergados, "An IMS-based network architecture for WiMAX-UMTS and WiMAX-WLAN Interworking”, Computer Communications, 34 (2011) pp. 1077-1099.

[2] Fei Shi, Keqiu Li, Yanming Shen, "Seamless handoff scheme in Wi-Fi and WiMAX heterogeneous networks", Future Generation Computer Systems, 26 (2012) pp 1403-1408.

[3] Xu Haibo, Tian Hui, Zhang Ping, "A novel terminal-controlled handover scheme in heterogeneous wireless networks", Computers and Electrical Engineering 36 (2012) pp 269-279.

[4] Ali Çalhan \& Celal Çeken, "An Adaptive Neuro-Fuzzy Based Vertical Handoff Decision Algorithm for Wireless Heterogeneous Networks" proceedings of 21st Annual IEEE International symposium on Personal, Indoor and Mobile Radio Communications, 2010, pp 2271-2276.

[5] Manoj Sharma, Dr. R.K. Khola “An Intelligent Handover Decision System for Multi Network Environment” Journal of Electrical \& Electronics Engineering Vol. 9, No. 2, 2013, pp 1067-1072.

[6] Kaveh Shafiee, Alireza Attar, Victor C. M. Leung, "Optimal Distributed Vertical Handoff Strategies in Vehicular Heterogeneous Networks", IEEE Journal On Selected Areas In Communications, Vol. 29, No. 3, March 2011, Pp 534-544.

[7] Manoj Sharma, Dr. R.K. Khola, "An Intelligent Approach for Handover Decision in Heterogeneous Wireless Environment”, International Journal of Engineering Vol.4, No. 5, 2016, pp 452-462.

[8] Shih-Jung Wu, "Fuzzy-Based Handover Decision Scheme for Next-Generation Heterogeneous Wireless Networks", Journal of Convergence Information Technology, Volume 6, Number 4. April 2016, pp 285-297.

[9] Manoj Sharma, Dr. R.K. Khola, "Pre Decision Based Handoff in Multi Network Environment" Advances in Intelligent and Soft Computing Vol.167, 2012, pp 609-616. D.O.I 10.1007/978-3-64230111-7_58. (A Springer Berlin Heidelberg Publication).

[10] W. Zhang, Handover decision using fuzzy MADM heterogeneous networks, IEEE Wireless Commun and Networking Conference, WCNC., Atlanta, USA, 2:653- 658, 2013

[11] Manoj Sharma, Dr. R.K Khola "Intelligent Approach for Seamless Mobility in Multi Network Environment," International Journal Communication Vol. 2, No. 2, July 2015, pp 43-47.

[12] Liu Xia, Ling-ge Jiang and Chen He, "A Novel Fuzzy Logic Vertical Handoff Algorithm with Aid of Differential prediction and Pre- Decision Method", IEEE National Conference on Communications 24-28 June 2015, pp 5665 - 5670.

[12] [13] Pramod Goyal, D. K. Lobiyal, C. P. Katti, "Vertical handoff in heterogeneous wireless networks", IEEE 2017 International Conference on Computing, Communication and Automation (ICCCA), India, 5-6 May 2017.

[14] I. Kustiawan, Tommi Hariyadi, D. R. Nurjannah, "Vertical handoff model in next generation wireless networks", IOP Conference Series: Materials Science and Engineering, Indonesia, May 2020. 